\title{
Stereotactic radiosurgery for secretory pituitary adenomas: systematic review and International Stereotactic Radiosurgery Society practice recommendations
}

\author{
David Mathieu, MD, ${ }^{1}$ Rupesh Kotecha, MD, ${ }^{2}$ Arjun Sahgal, MD, ${ }^{3}$ Antonio De Salles, MD, PhD, ${ }^{4}$ \\ Laura Fariselli, MD, ${ }^{5}$ Bruce E. Pollock, MD, ${ }^{6}$ Marc Levivier, MD, PhD, ${ }^{7}$ Lijun Ma, PhD, ${ }^{8}$ \\ lan Paddick, MSc, ${ }^{9}$ Jean Regis, MD, PhD, ${ }^{10,11}$ Shoji Yomo, MD, PhD, ${ }^{12}$ John H. Suh, MD, ${ }^{13}$ \\ Muni Rubens, PhD, ${ }^{2}$ and Jason P. Sheehan, MD, PhD ${ }^{14}$ \\ 'Division of Neurosurgery, Université de Sherbrooke, Centre de recherche du Centre Hospitalier Universitaire de Sherbrooke, \\ Sherbrooke, Quebec, Canada; 'Department of Radiation Oncology, Miami Cancer Institute, Baptist Health South Florida, Miami, \\ Florida; ${ }^{3}$ Department of Radiation Oncology, Sunnybrook Health Sciences Centre, University of Toronto, Ontario, Canada; \\ ${ }^{4}$ Department of Neurosurgery, University of California, Los Angeles, California; ${ }^{5}$ Radiotherapy Unit-Neurosurgery Department, \\ Fondazione IRCCS Istituto Neurologico Carlo Besta, Milan, Italy; ${ }^{\circ}$ Department of Neurologic Surgery, Mayo Clinic, Rochester, \\ Minnesota; ${ }^{7}$ Department of Neurosurgery and Gamma Knife Center, Lausanne University Hospital, Lausanne, Switzerland; \\ ${ }^{8}$ Department of Radiation Oncology, University of California, San Francisco, California; ${ }^{9}$ Medical Physics Ltd., Reading, United \\ Kingdom; ${ }^{10} \mathrm{Cromwell} \mathrm{Hospital,} \mathrm{London,} \mathrm{United} \mathrm{Kingdom;}{ }^{11} \mathrm{Functional}$ and Stereotaxic Neurosurgery Department, Clinical \\ Neuroscience Federation, La Timone Hospital, Aix-Marseille University, Marseille, France; ${ }^{2}$ Division of Radiation Oncology, Aizawa \\ Comprehensive Cancer Center, Aizawa Hospital, Matsumoto, Japan; ${ }^{13}$ Department of Radiation Oncology, Taussig Cancer Institute, \\ Cleveland Clinic, Cleveland, Ohio; and ${ }^{14}$ Department of Neurological Surgery, University of Virginia, Charlottesville, Virginia
}

OBJECTIVE A systematic review was performed to provide objective evidence on the use of stereotactic radiosurgery (SRS) in the management of secretory pituitary adenomas and develop consensus recommendations.

METHODS The authors performed a systematic review of the English-language literature up until June 2018 using the PRISMA guidelines. The PubMed (Medline), Embase, and Cochrane databases were searched. A total of 45 articles reporting single-institution outcomes of SRS for acromegaly, Cushing's disease, and prolactinomas were selected and included in the analysis.

RESULTS For acromegaly, random effects meta-analysis estimates for crude tumor control rate, crude endocrine remission rate, and any new hypopituitarism rates were $97.0 \%$ (95\% $\mathrm{Cl} 96.0 \%-98.0 \%), 44.0 \%$ (95\% $\mathrm{Cl} 35.0 \%-53.0 \%$ ), and $17.0 \%$ (95\% Cl 13.0\%-23.0\%), respectively. For Cushing's disease, random effects estimates for crude tumor control rate, crude endocrine remission rate, and any new hypopituitarism rate were $92.0 \%(95 \% \mathrm{Cl} 87.0 \%-95.0 \%), 48.0 \%$ (95\% Cl 35.0\%-61.0\%), and 21.0\% (95\% Cl 13.0\%-31.0\%), respectively. For prolactinomas, random effects estimates for crude tumor control rate, crude endocrine remission rate, and any new hypopituitarism rate were $93.0 \%(95 \% \mathrm{Cl}$ $90.0 \%-95.0 \%$ ), $28.0 \%$ (95\% Cl 19.0\%-39.0\%), and 12.0\% (95\% Cl 6.0\%-24.0\%), respectively. Meta-regression analysis did not show a statistically significant association between mean margin dose with crude endocrine remission rate or mean margin dose with development of any new hypopituitarism rate for any of the secretory subtypes.

CONCLUSIONS SRS offers effective tumor control of hormone-producing pituitary adenomas in the majority of patients but a lower rate of endocrine improvement or remission.

https://thejns.org/doi/abs/10.3171/2021.2.JNS204440

KEYWORDS acromegaly; Cushing's disease; prolactinomas; secretory pituitary adenomas; pituitary surgery; stereotactic radiosurgery

ABBREVIATIONS ACTH = adrenocorticotrophic hormone; GH = growth hormone; IGF-1 = insulin-like growth factor-1; ISRS = International Stereotactic Radiosurgery Society; LINAC = linear accelerator; NFA = nonfunctioning adenoma; OGTT = oral glucose tolerance test; PA = pituitary adenoma; PRL = prolactin; RT = radiation therapy; SRS = stereotactic radiosurgery.

SUBMITTED December 27, 2020. ACCEPTED February 19, 2021

INCLUDE WHEN CITING Published online September 3, 2021; DOI: 10.3171/2021.2.JNS204440. 
$\mathrm{P}$ TUITARY adenomas (PAs) are common benign neoplastic lesions arising from cells of the anterior pituitary gland. Approximately $70 \%$ of PAs are associated with hormonal hypersecretion syndromes, among which the most common are tumors secreting prolactin (PRL), growth hormone (GH), or adrenocorticotrophic hormone (ACTH). ${ }^{1}$ The management of secretory PA represents a significant challenge because of the need to adequately control hormonal hypersecretion in addition to tumor growth. Management options for secretory PA include medical therapy, surgical resection, and radiation therapy (RT), alone or in combination. Stereotactic radiosurgery (SRS) is being increasingly used whenever possible as an alternative to fractionated RT, with the goal of limiting radiation exposure to adjacent normal tissues. However, the literature regarding the use of SRS in the treatment of PAs comprises only multiple retrospective studies. This study was performed under the International Stereotactic Radiosurgery Society (ISRS) guideline project to systematize the available literature and develop consensus recommendations to guide the care of patients afflicted by the most common secretory subtypes: prolactinomas, acromegaly (GH secreting), and Cushing's disease (ACTH secreting).

\section{Methods}

\section{Selection of Articles}

Using the PRISMA guidelines, ${ }^{2}$ a systematic review of the English-language literature up until June 2018 was performed. The PubMed (Medline), Embase, and Cochrane databases were searched for relevant articles using the following MeSH terms: (Gamma Knife OR Radiosurgery OR LINAC OR CyberKnife) AND (pituitary adenoma OR Cushing's disease OR acromegaly OR prolactinoma). The initial search provided 1045 articles whose titles and abstracts were screened for relevance, with 134 articles retained at this stage. Full-text screening was performed by using the following inclusion criteria: single-institution study, more than 10 patients reported, and both tumor and endocrine control data reported. Studies reporting the use of proton-based SRS or fractionated stereotactic RT and studies only reporting Nelson syndrome cases were excluded. In cases where multiple studies were from the same institution, only the most recent relevant publication with the most extensive data was included. After full-text screening, a total of 45 articles were selected for the final analyses. All studies were retrospective and reported low-quality evidence. Many studies reported pooled outcomes of all PAs treated at the reporting institution. From those, the data reporting the outcomes of Cushing's disease, acromegaly, or prolactinoma were extracted, and the data were extracted separately for those three different subtypes. Separate analyses were performed for each secretory subtype. The final review included 28 acromegaly studies, 13 Cushing's disease studies, and 13 prolactinoma studies. Figure 1 describes the search strategy and inclusion methodology as a PRISMA flow diagram.

\section{Outcome Measures and Statistical Analyses}

The primary outcomes of the study were endocrine re- mission off medication and tumor control rates for each of the 3 main secretory subtypes. Endocrine and cranial nerve morbidity was also assessed. Data collection included the number of cases per study, radiosurgery device used, mean follow-up time, prior resection, mean margin radiation dose and dose range, mean tumor volume, local control rate, endocrine remission and relapse rates, time to remission, criteria used for endocrine remission definition, predictors of endocrine response, new or worsening pituitary insufficiency rates, and new or worsening visual or cranial nerve morbidity.

Statistical analyses were performed using R Studio (version 1.1.423). Meta-analyses, tests for heterogeneity, analysis of publication bias, and meta-regressions were done with $\mathrm{R}$ package metafor (version 2.0-0). Study variances for the overall estimates and for meta-regression were calculated using the DerSimonian-Laird method. Weighted random effects models were used to calculate pooled estimates for crude rates of local control, endocrine remission rate, and new-onset hypopituitarism for each of the secretory subtypes. Given the study population variances across the studies, and since each study involves treatment decisions, the random effects model was considered superior to the fixed effects model when calculating pooled estimates. The $\mathrm{I}^{2}$ statistic was used for identifying heterogeneity: $\mathrm{I}^{2}$ values of $0 \%, 25 \%, 50 \%$, and $75 \%$ were interpreted as indicating absent, low, moderate, and high heterogeneity, respectively. Funnel plots and the Egger test ( $\mathrm{p}$ value $<0.05$ indicating presence of bias) were used for identifying publication bias. Meta-regression analyses were performed to identify potential associations between endocrine remission rate or development of new-onset hypopituitarism and mean margin dose.

\section{Results}

\section{Acromegaly}

A total of 28 studies reporting acromegaly cases met inclusion criteria and were evaluated in the final analysis (Supplementary Table 1). All studies were retrospective and provided low-quality evidence. Selected articles were published between 1998 and 2018. The majority of studies reported patients treated by Gamma Knife radiosurgery (21 studies). ${ }^{3-23}$ CyberKnife was used in 2 studies, ${ }^{24,25}$ linear accelerator (LINAC)-based SRS in 4 studies, ${ }^{26-29}$ and a rotating gamma unit (MASEP) in 1 study. ${ }^{30} \mathrm{~A}$ total of 1335 patients were included in the meta-analysis. SRS was usually performed for recurrence or adjuvant treatment after tumor resection $(65 \%-100 \%$ prior tumor resection in all except 2 studies). In single-fraction studies, mean margin doses reported ranged from 13.2 to 35 Gy. Reported tumor control rates varied between $94 \%$ and $100 \%$, and new neurological or visual deficit rates ranged between $0 \%$ and $11 \%$. Crude endocrine remission rates varied between $0 \%$ and $82 \%$. However, there was variability among studies relative to the criteria used to define endocrine cure. Only one study used the current consensus definition (GH level after an oral glucose tolerance test [OGTT] less than $0.4 \mathrm{ng} / \mathrm{ml}$ and normalization of age- and sex-adjusted insulin-like growth factor-1 [IGF-1] levels). ${ }^{31}$ The most common criteria to define re- 


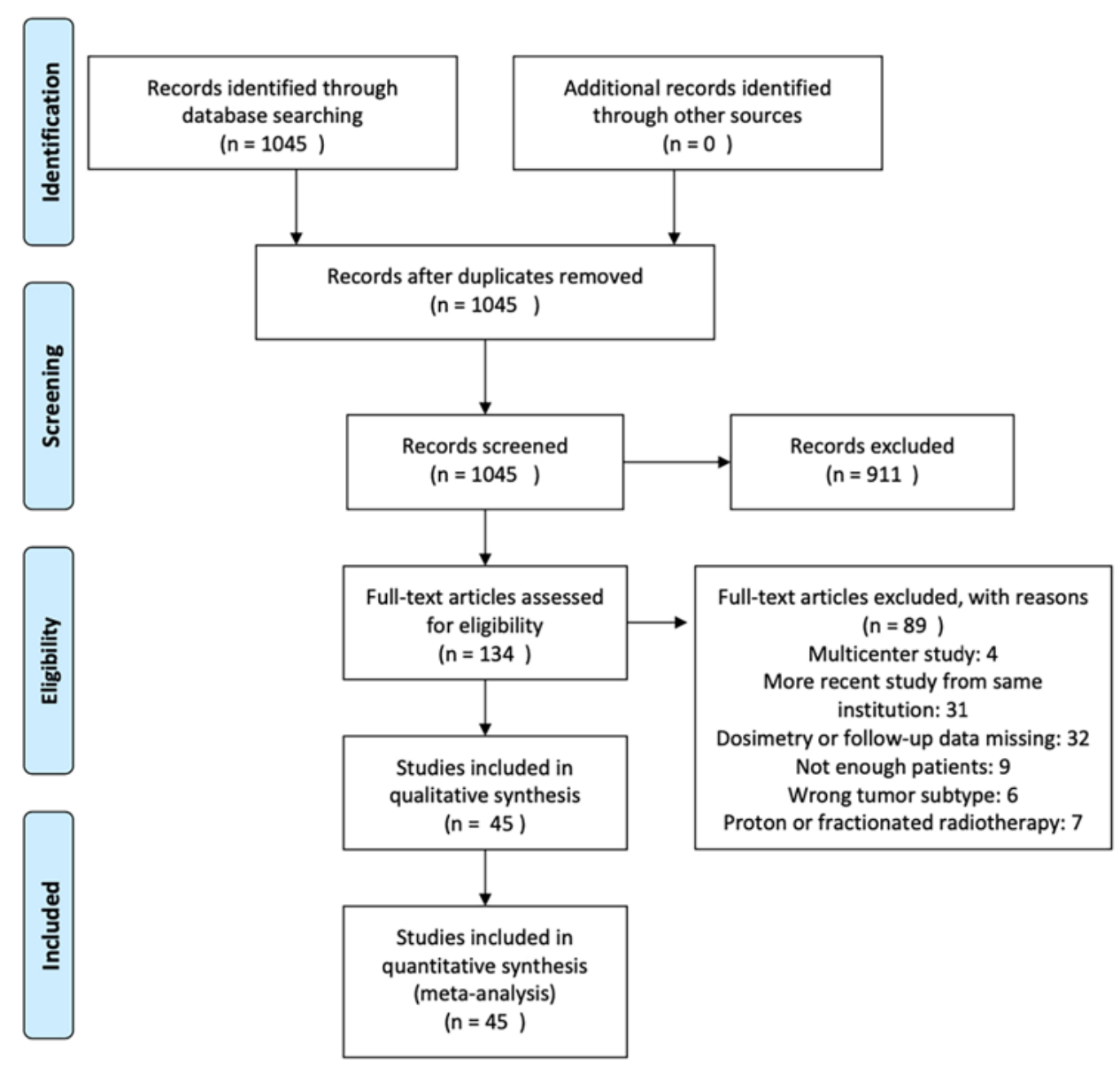

FIG. 1. PRISMA flow diagram demonstrating the search strategy for study selection and inclusion in the systematic review for secretory PA SRS. Figure is available in color online only.

mission were normalization of age- and sex-adjusted IGF1 levels and random $\mathrm{GH}$ values less than $2-2.5 \mathrm{ng} / \mathrm{ml}$, used in 10 studies $(35.7 \%)$. New or worsened hypopituitarism was reported in $0 \%-40 \%$ of treated patients. The following factors were identified by some studies as being predictors of endocrine remission: GH and IGF-1 levels less than 2.25 times normal at time of SRS, , ,8,14,17 absence of cavernous sinus invasion, ${ }^{8,11}$ male sex, ${ }^{11}$ higher margin dose, ${ }^{21}$ and absence of suppressive medication at SRS..$^{14}$

Random effects meta-analyses for crude tumor control, crude endocrine remission, and any new hypopituitarism are shown in Figs. 2-4, with random effects estimates of $97.0 \%$ (95\% CI $96.0 \%-98.0 \%$ ), $44.0 \%$ (95\% CI 35.0\%$53.0 \%$ ), and $17.0 \%$ (95\% CI $13.0 \%-23.0 \%$ ), respectively. Publication bias was assessed by using the Egger test. No evidence of publication bias was found for the studies included for assessing crude tumor control rate $(\mathrm{p}=0.251)$ and crude endocrine remission rate $(\mathrm{p}=0.782)$. However, publication bias was evident for studies included for assessing new hypopituitarism rate $(\mathrm{p}=0.039)$. Funnel plots for publication bias are shown in Supplementary Fig. 1. Supplementary Fig. 2 shows meta-regression plots for association of mean margin dose with crude endocrine remission rate and any new hypopituitarism rate. Metaregression analysis did not show a statistically significant association between mean margin dose with crude endocrine remission rate $(\mathrm{p}=0.054)$ and mean margin dose with any new hypopituitarism rate $(\mathrm{p}=0.685)$.

\section{Cushing's Disease}

A total of 13 studies reporting Cushing's disease cases met inclusion criteria and were evaluated in the final analysis (Supplementary Table 2). All studies were retrospective and provided low-quality evidence. Selected articles were published between 1998 and 2018. Eight studies reported patients treated by Gamma Knife radiosurgery, ${ }^{4,12}$, 20,32-36 whereas LINAC-based SRS was used in 4 studies $^{29,37-39}$ and a rotating gamma unit (MASEP) in 1 study. ${ }^{30}$ A total of 416 patients were included in the meta-analysis. SRS was usually performed for recurrence or adjuvant treatment after tumor resection $(57 \%-100 \%$ prior tumor resection in all except 1 study). The mean margin dose reported ranged from 14.7 to $35 \mathrm{~Gy}$. Reported tumor control rates varied between $83 \%$ and $100 \%$, and new neurological or visual deficit rates ranged between $0 \%$ and $17 \%$. Crude endocrine remission rates varied between $6 \%$ and $81 \%$. There was variability among studies relative to the criteria used to define endocrine cure, with the most common criteria used being normalization of 24-hour urinary 


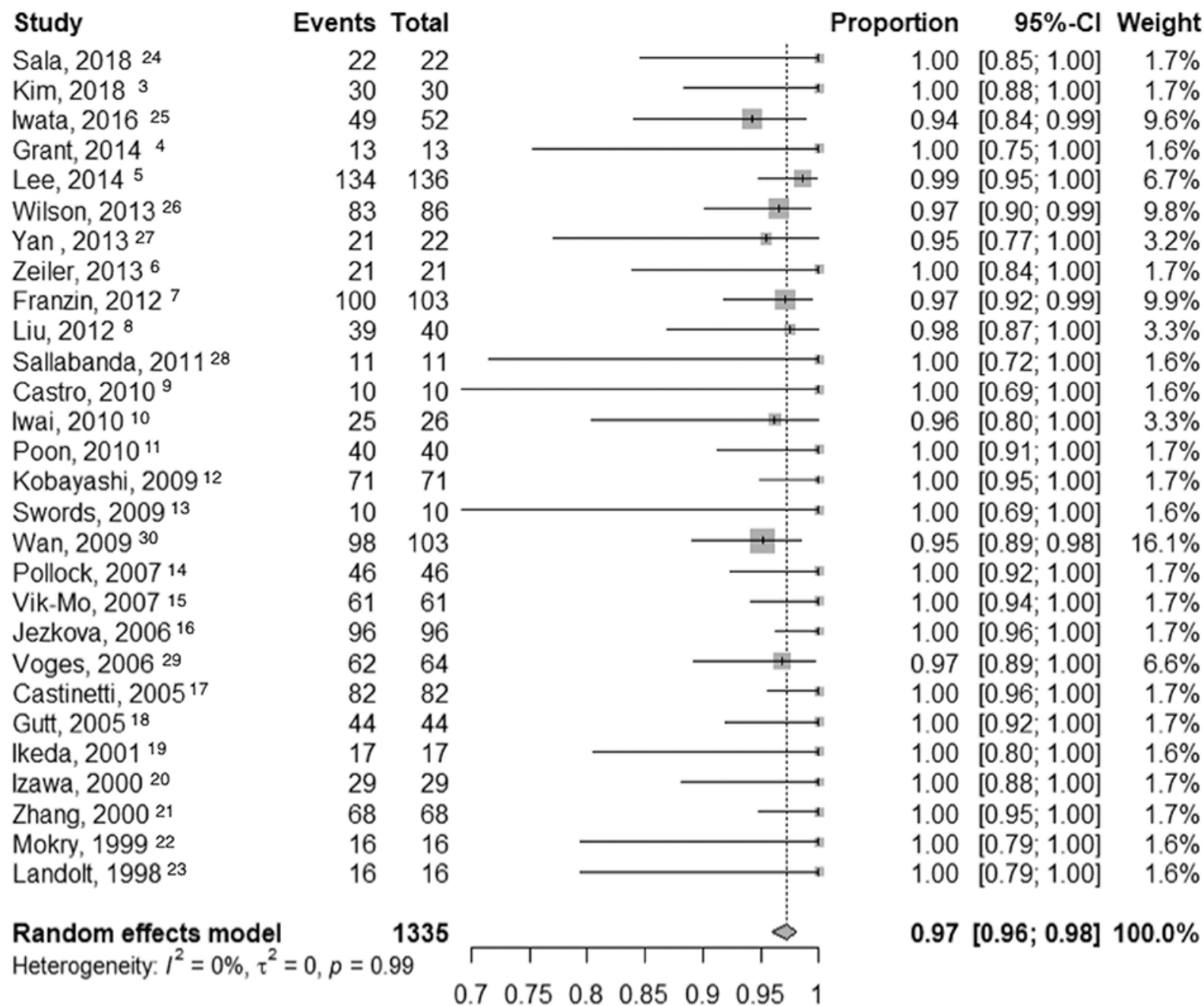

FIG. 2. Forest plot for crude tumor control rate for acromegaly. Squares indicate the proportions from individual studies and horizontal lines indicate the $95 \% \mathrm{Cl}$. The size of the data marker corresponds to the relative weight assigned in the pooled analysis using the random effects model. The diamond indicates the pooled proportion with $95 \% \mathrm{Cl}$.

free cortisol in 5 studies (38.5\%). New or worsened hypopituitarism was reported in $0 \%-50 \%$ of treated patients. The following factors were identified by some studies as being predictors of endocrine remission: smaller target volume, ${ }^{36}$ lower urinary cortisol level at SRS, ${ }^{33}$ and $a b-$ sence of suppressive medication at SRS. ${ }^{34,36}$

Random effects meta-analyses for crude tumor control, crude endocrine remission, and any new hypopituitarism are shown in Fig. 5, with random effects estimates of $92.0 \%$ (95\% CI $87.0 \%-95.0 \%$ ), $48.0 \%$ (95\% CI $35.0 \%-$ $61.0 \%$ ), and $21.0 \%$ (95\% CI $13.0 \%-31.0 \%$ ), respectively. Publication bias was assessed by using the Egger test. No evidence of publication bias was found for the studies included for assessing crude tumor control rate $(p=0.198)$ and crude endocrine remission rate $(\mathrm{p}=0.524)$. However, publication bias was evident for studies included for assessing new hypopituitarism rate $(\mathrm{p}=0.023)$. Funnel plots for publication bias are shown in Supplementary Fig. 3. Supplementary Fig. 4 shows meta-regression plots for association of mean margin dose with crude endocrine remission rate and any new hypopituitarism rate. Metaregression analysis did not show a statistically significant association between mean margin dose with crude endocrine remission rate $(p=0.563)$ or mean margin dose with any new hypopituitarism rate $(\mathrm{p}=0.952)$.

\section{Prolactinomas}

A total of 13 studies reporting prolactinoma cases met inclusion criteria and were evaluated in the final analysis (Supplementary Table 3). All studies were retrospective and provided low-quality evidence. Selected articles were published between 1998 and 2018. Nine studies reported patients treated by Gamma Knife radiosurgery, $12,20,22,40-45$ whereas LINAC-based SRS was used in 3 studies, ${ }^{29,46,47}$ and a rotating gamma unit (MASEP) in 1 study. ${ }^{30} \mathrm{~A}$ total of 538 patients were included in the meta-analysis. SRS was performed after prior tumor resection in $2 \%-100 \%$ of cases for patients who had tumor growth and persistent hyperprolactinemia on medical therapy, or who were 


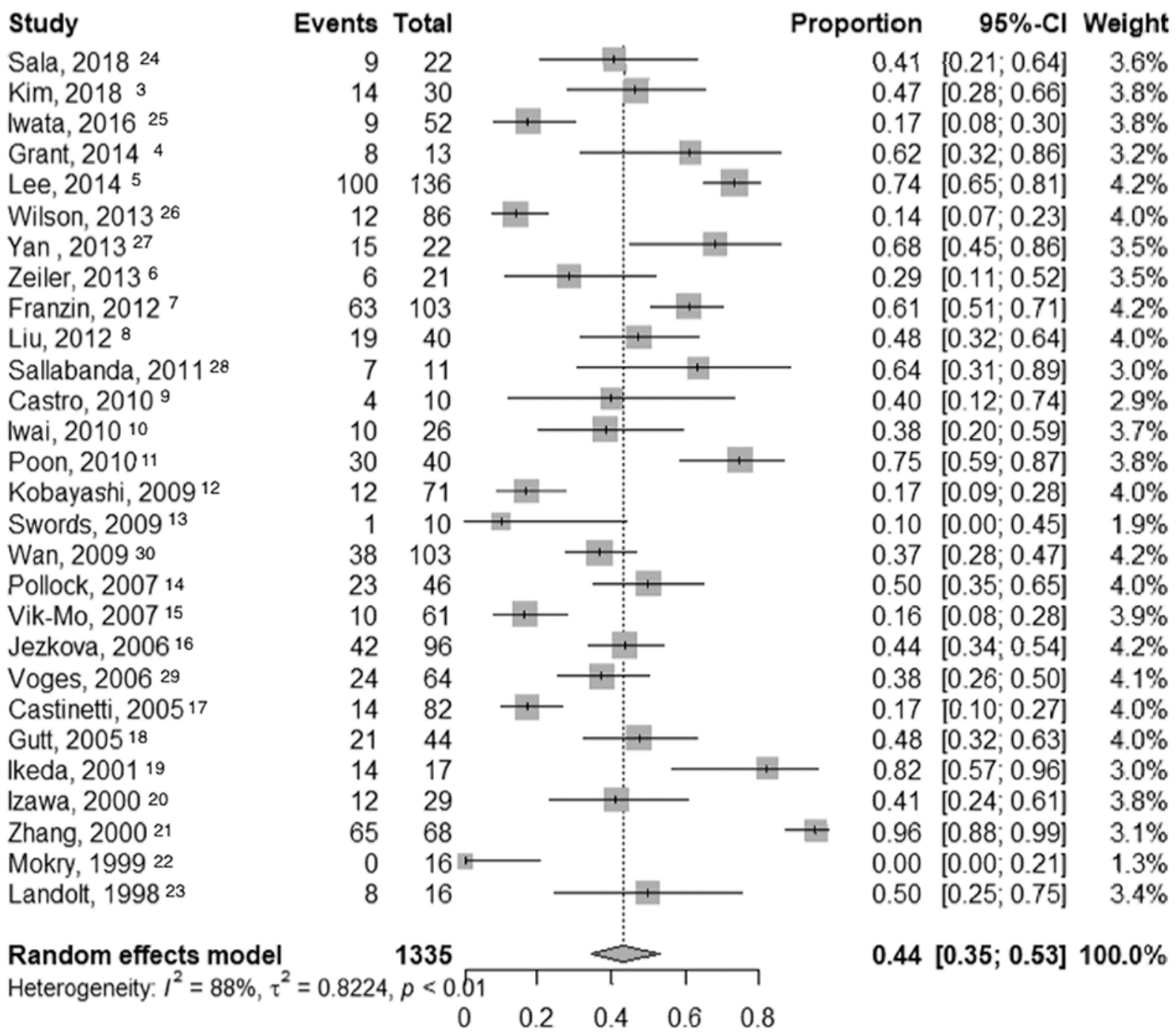

FIG. 3. Forest plot for crude endocrine remission rate for acromegaly. Squares indicate the proportions from individual studies and horizontal lines indicate the $95 \% \mathrm{Cl}$. The size of the data marker corresponds to the relative weight assigned in the pooled analysis using the random effects model. The diamond indicates the pooled proportion with $95 \% \mathrm{Cl}$.

intolerant to medication. The mean margin dose reported ranged from 13.5 to $35 \mathrm{~Gy}$. Reported tumor control rates varied between $86 \%$ and $100 \%$, and new neurological or visual deficit rates ranged between $0 \%$ and $5 \%$. Crude endocrine remission rates varied between $6 \%$ and $81 \%$. There was variability among studies relative to the criteria used to define endocrine cure, with most (8 studies, $61.5 \%$ ) only stating normal PRL levels without specifying any numeral value or if patients needed to be off antisecretory medication. New or worsened hypopituitarism was reported in $0 \%-62 \%$ of treated patients. The following factors were identified by some studies as being predictors of endocrine remission: tumor volume less than $3 \mathrm{ml}{ }^{41}$ PRL levels less than $200 \mu \mathrm{g} / \mathrm{L},{ }^{41}$ and margin dose more than $30 \mathrm{~Gy}{ }^{45}$ None of the included studies reported the use of a dopamine agonist at SRS as a significant predictor of endocrine outcome.

Random effects meta-analyses for crude tumor control, crude endocrine remission, and any new hypopituitarism are shown in Fig. 6, with random effects estimates of 93.0\% (95\% CI 90.0\%-95.0\%), $28.0 \%$ (95\% CI $19.0 \%-$ $39.0 \%$ ), and $12.0 \%$ (95\% CI $6.0 \%-24.0 \%$ ), respectively. Publication bias was assessed by using the Egger test. No evidence of publication bias was found for the studies included for assessing crude endocrine remission rate $(\mathrm{p}=$ $0.783)$ and new hypopituitarism rate $(\mathrm{p}=0.222)$. However, publication bias was evident for studies included for assessing crude tumor control rate $(\mathrm{p}=0.021)$. Funnel plots for publication bias are shown in Supplementary Fig. 5. Supplementary Fig. 6 shows meta-regression plots for association of mean margin dose with crude endocrine remission rate and any new hypopituitarism rate. Metaregression analysis did not show a statistically significant association of mean margin dose with crude endocrine remission rate $(p=0.699)$ or mean margin dose with any new hypopituitarism rate $(\mathrm{p}=0.986)$. 


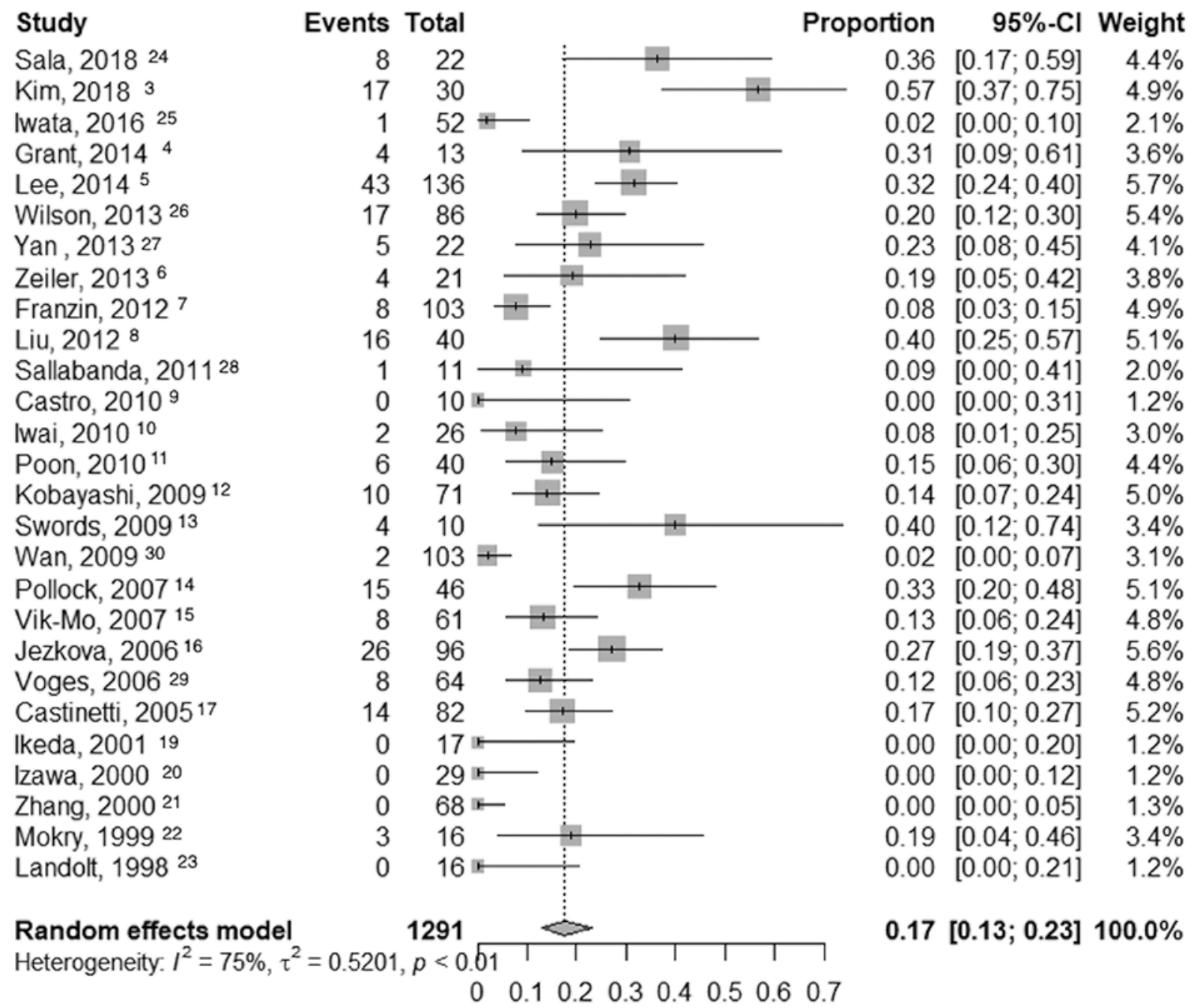

FIG. 4. Forest plot for any new hypopituitarism rate for acromegaly. Squares indicate the proportions from individual studies and horizontal lines indicate the $95 \% \mathrm{Cl}$. The size of the data marker corresponds to the relative weight assigned in the pooled analysis using the random effects model. The diamond indicates the pooled proportion with $95 \% \mathrm{Cl}$.

\section{Discussion}

SRS is widely used in the management of PAs, both nonsecretory and secretory. An ISRS-performed systematic review of the literature and consensus guidelines for nonfunctioning adenomas (NFAs) of the pituitary have recently been published. ${ }^{48}$ Whereas the goal of treatment for NFAs is limited to tumor growth control, there is an additional challenge for secretory adenomas, namely control of the associated hypersecretory syndrome. For this systematic review, we selected 45 studies that reported the outcomes of SRS for patients with secretory PA. There are no prospective studies that have addressed this topic, to our knowledge, and most papers are single-center retrospective cohort studies and thus are evidence level IV studies. Nevertheless, there are still significant findings to guide patient management and formulate recommendations (Table 1).

\section{Tumor Growth Control}

SRS has been mostly used in an adjuvant or recurrent setting after prior tumor resection. All tumor types seem to demonstrate excellent crude local tumor control after SRS. Based on our meta-analysis, tumor control at last follow-up can be expected in $97 \%$ of acromegaly patients, 92\% of Cushing's disease patients, and $93 \%$ of prolactinoma patients. These rates are similar to those reported in NFA patients. ${ }^{48}$ However, there are a number of limitations that need to be taken into consideration when interpreting these results. First, most studies reported only crude control rates, and not actuarial time-dependent control estimates. Long-term follow-up could reveal late tumor regrowth, thus lowering control rates. In addition, the majority of investigators did not differentiate between actual tumor regression or stability in tumor size after SRS. While this might not make a difference from a strictly neuro-oncological point of view, this distinction remains important and should be reported in future studies to better assess if tumor size variation after SRS has any impact on endocrine outcome. Finally, none of the investigators reported using volumetric evaluation for assessment of tu- 
A Study

$2018^{32}$

Marek, $2015^{30}$

Grant, $2014^{4}$

Wilson, 2014 ${ }^{37}$

Sheehan, $2013^{34}$

Wein, 2012

Kobayashi, $2009^{12}$

Wan, $2009^{30}$

Tinnel, $2008^{35}$

Castinetti, $2007^{36}$

Voges, $2006^{20}$

Devin, $2004^{30}$

Izawa, $2000^{20}$

Random effects model

Heterogeneity: $I^{2}=23 \%, \tau^{2}=0.1673, p=0.22$
Events Total

$11 \quad 12$

$15 \quad 15$

$30 \quad 36$

$94 \quad 96$

$\begin{array}{ll}17 & 17\end{array}$

$30 \quad 30$

6168

10

$40 \quad 40$

$\begin{array}{ll}15 & 17\end{array}$

$32 \quad 35$

10

12

416 -
$26 \quad 26$

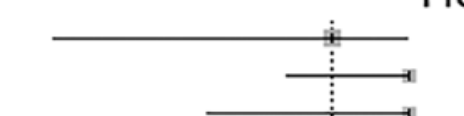

Proportion $\quad 95 \%-\mathrm{Cl}$ Weight

$0.92[0.62 ; 1.00] \quad 4.9 \%$

$1.00[0.87 ; 1.00] \quad 2.8 \%$

$1.00[0.78 ; 1.00] \quad 2.8 \%$

$0.83[0.67 ; 0.94] \quad 16.8 \%$

$0.98[0.93 ; 1.00] \quad 9.1 \%$

$1.00[0.80 ; 1.00] \quad 2.8 \%$

$1.00[0.88 ; 1.00] \quad 2.8 \%$

$0.90[0.80 ; 0.96] \quad 18.9 \%$

$0.83[0.52 ; 0.98] \quad 8.1 \%$

$1.00[0.91 ; 1.00] \quad 2.8 \%$

$0.88[0.64 ; 0.99] \quad 8.4 \%$

$0.91[0.77 ; 0.98] \quad 11.6 \%$

$0.83[0.52 ; 0.98] \quad 8.1 \%$

$0.92[0.87 ; 0.95] 100.0 \%$
B

Study

Narayan, $2018^{32}$

Marek, $2015^{30}$

Grant, $2014^{4}$

Wilson, $2014^{37}$

Sheehan, $2013^{34}$

Wein, $2012{ }^{36}$

Kobayashi, $2009^{12}$

Wan, $2009^{30}$

Tinnel, $2008{ }^{35}$

Castinetti, $20077^{36}$

Voges, $2006^{20}$

Devin, $2004^{30}$

Izawa, $2000^{20}$

Random effects model

Heterogeneity: $I^{2}=81 \%, \tau^{2}=0.6844, p<0.01$
Events Total

812

$\begin{array}{ll}21 & 15 \\ 11 & 26\end{array}$

236

$67 \quad 96$

$\begin{array}{ll}10 & 17\end{array}$

1130

1968

$\begin{array}{ll}6 & 12\end{array}$

$17 \quad 40$

$\begin{array}{ll}9 & 17\end{array}$

$17 \quad 35$

2

12

416

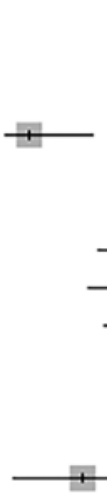

$\begin{array}{lllll}0.6 & 0.7 & 0.8 & 0.9 & 1\end{array}$

$\begin{array}{rrr}\text { Proportion } & 95 \%-C l & \text { Weight } \\ 0.67 & {[0.35 ; 0.90]} & 6.7 \% \\ 0.81 & {[0.61 ; 0.93]} & 7.6 \% \\ 0.73[0.45 ; 0.92] & 6.9 \% \\ 0.06[0.01 ; 0.19] & 5.8 \% \\ 0.70[0.60 ; 0.79] & 9.6 \% \\ 0.59[0.33 ; 0.82] & 7.6 \% \\ 0.37[0.20 ; 0.56] & 8.5 \% \\ 0.28[0.18 ; 0.40] & 9.3 \% \\ 0.50[0.21 ; 0.79] & 6.9 \% \\ 0.42[0.27 ; 0.59] & 9.0 \% \\ 0.53[0.28 ; 0.77] & 7.7 \% \\ 0.49[0.31 ; 0.66] & 8.8 \% \\ 0.17[0.02 ; 0.48] & 5.5 \%\end{array}$

$0.48[0.35 ; 0.61] 100.0 \%$

\section{Study}

Narayan, $2018^{32}$

Marek, $2015^{30}$

Grant, $2014^{4}$

Wilson, $2014^{37}$

Sheehan, $2013^{34}$

Wein, $2012^{38}$

Kobayashi, $2009^{12}$

Wan, $2009^{30}$

Tinnel, $2008{ }^{35}$

Castinetti, $2007^{36}$

Voges, $2006^{20}$

Devin, $2004^{39}$

Izawa, $2000^{20}$

Random effects model $\quad 416$

Events Total

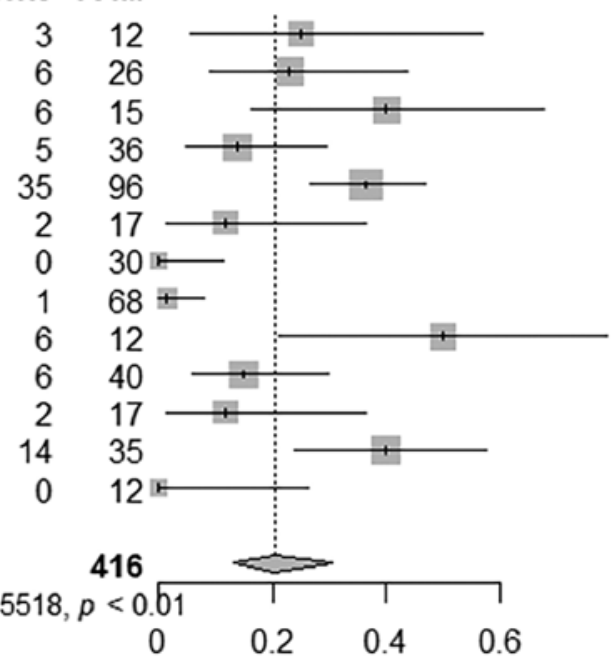

Proportion $\quad 95 \%-\mathrm{Cl}$ Weight

$0.25[0.05 ; 0.57] \quad 7.4 \%$

$0.23[0.09 ; 0.44] \quad 9.6 \%$

$0.40[0.16 ; 0.68] \quad 8.9 \%$

$0.14[0.05 ; 0.29] \quad 9.4 \%$

$0.36[0.27 ; 0.47] \quad 12.3 \%$

$0.12[0.01 ; 0.36] \quad 6.6 \%$

$0.00[0.00 ; 0.12] \quad 2.8 \%$

$0.01[0.00 ; 0.08] \quad 4.7 \%$

$0.50[0.21 ; 0.79] \quad 8.3 \%$

$0.15[0.06 ; 0.30] \quad 9.8 \%$

$0.12[0.01 ; 0.36] \quad 6.6 \%$

$0.40[0.24 ; 0.58] \quad 11.0 \%$

$0.00[0.00 ; 0.26] \quad 2.8 \%$

$0.21[0.13 ; 0.31] 100.0 \%$

FIG. 5. Forest plot for crude tumor control rate, crude endocrine remission rate, and any new hypopituitarism rate for Cushing's disease. Squares indicate the proportions from individual studies and horizontal lines indicate the $95 \% \mathrm{Cl}$. The size of the data marker corresponds to the relative weight assigned in the pooled analysis using the random effects model. The diamond indicates the pooled proportion with $95 \% \mathrm{Cl}$. A: Forest plot for crude tumor control rate. B: Forest plot meta-analysis for crude endocrine remission rate. C: Forest plot for any new hypopituitarism rate. 


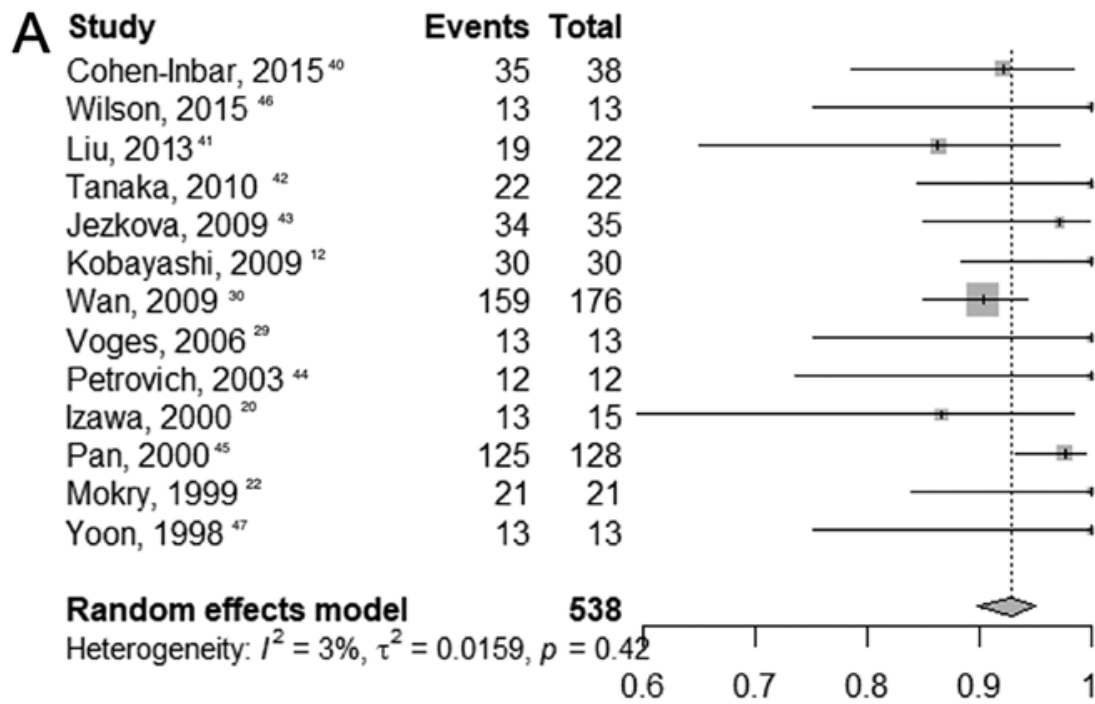

$\begin{array}{rrr}\text { Proportion } & \mathbf{9 5 \%} \text {-Cl } & \text { Weight } \\ 0.92 & {[0.79 ; 0.98]} & 10.1 \% \\ 1.00 & {[0.75 ; 1.00]} & 1.8 \% \\ 0.86 & {[0.65 ; 0.97]} & 9.5 \% \\ 1.00 & {[0.85 ; 1.00]} & 1.8 \% \\ 0.97 & {[0.85 ; 1.00]} & 3.6 \% \\ 1.00 & {[0.88 ; 1.00]} & 1.9 \% \\ 0.90 & {[0.85 ; 0.94]} & 47.0 \% \\ 1.00 & {[0.75 ; 1.00]} & 1.8 \% \\ 1.00 & {[0.74 ; 1.00]} & 1.8 \% \\ 0.87 & {[0.60 ; 0.98]} & 6.4 \% \\ 0.98 & {[0.93 ; 1.00]} & 10.6 \% \\ 1.00 & {[0.84 ; 1.00]} & 1.8 \% \\ 1.00 & {[0.75 ; 1.00]} & 1.8 \%\end{array}$

\section{$0.93[0.90 ; 0.95] 100.0 \%$}

B Study

Cohen-Inbar, $2015^{40}$

Wilson, $2015^{46}$

Liu, $2013^{41}$

Tanaka, $2010^{42}$

Jezkova, $2009^{43}$

Kobayashi, $2009^{12}$

Wan, $2009^{30}$

Voges, $2006^{29}$

Petrovich, $2003^{4}$

Izawa, $2000{ }^{20}$

Pan, $2000^{45}$

Mokry, $1999^{22}$

Yoon, $1998{ }^{47}$

\section{Events Total}

1938

113

$\begin{array}{ll}1 & 22 \\ 4 & 22\end{array}$

$13 \quad 35$

$5 \quad 30$

$41 \quad 176$

213

1012

$\begin{array}{rr}3 & 15 \\ 27 & 128\end{array}$

$\begin{array}{rr}27 & 128 \\ 4 & 21\end{array}$

$11 \quad 13$

$0.7 \quad 0.8$

0.9

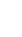

Proportion $\quad 95 \%-\mathrm{Cl}$ Weight

$0.50[0.33 ; 0.67] \quad 10.5 \%$

$0.00[0.00 ; 0.25] \quad 2.5 \%$

$0.05[0.00 ; 0.23] \quad 4.2 \%$

$0.18[0.05 ; 0.40] \quad 7.9 \%$

$0.37[0.21 ; 0.55] \quad 10.2 \%$

$0.17[0.06 ; 0.35] \quad 8.6 \%$

$0.23[0.17 ; 0.30] \quad 11.9 \%$

$0.15[0.02 ; 0.45] \quad 5.9 \%$

$0.83[0.52 ; 0.98] \quad 5.9 \%$

$0.20[0.04 ; 0.48] \quad 7.0 \%$

$0.21[0.14 ; 0.29] \quad 11.6 \%$

$0.19[0.05 ; 0.42] \quad 7.9 \%$

$0.85[0.55 ; 0.98] \quad 5.9 \%$

$0.28[0.19 ; 0.39] 100.0 \%$

Random effects model

Heterogeneity: $I^{2}=76 \%, \tau^{2}=0.5185, p<0$

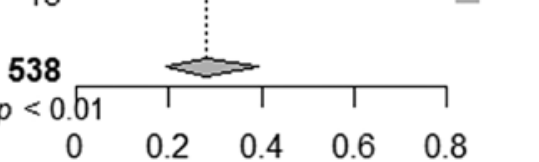

C Study

Cohen-Inbar, $2015^{40}$

Wilson, $2015^{46}$

Liu, 2013"1

Tanaka, $2010^{42}$

Jezkova, $2009^{43}$

Kobayashi, $2009^{12}$

Wan, $2009{ }^{30}$

Voges, $2006^{20}$

Petrovich, $2003^{44}$

Izawa, 2000 20

Pan, $2000^{45}$

Mokry, $1999^{22}$

Yoon, $1998{ }^{47}$

Random effects model

Heterogeneity: $I^{2}=80 \%, \tau^{2}=1.4965, p$

\section{Events Total}

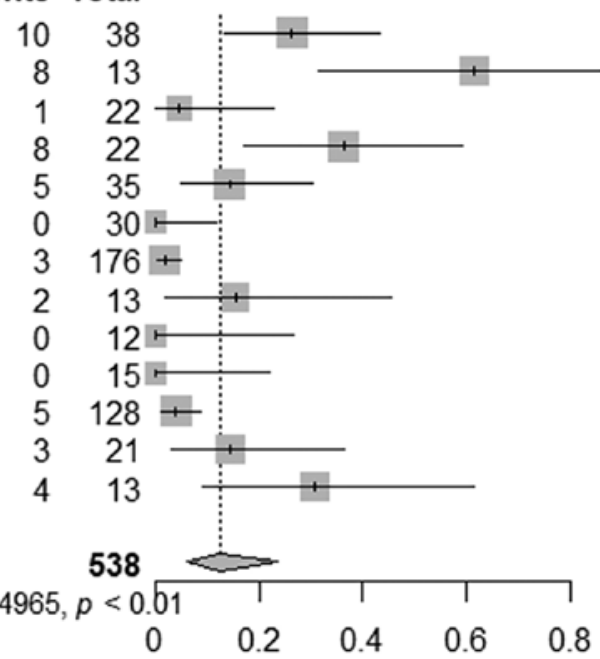

Proportion $\quad 95 \%-\mathrm{Cl}$ Weight

$0.26[0.13 ; 0.43] \quad 9.8 \%$

$0.62[0.32 ; 0.86] \quad 8.8 \%$

$0.05[0.00 ; 0.23] \quad 6.3 \%$

$0.36[0.17 ; 0.59] \quad 9.5 \%$

$0.14[0.05 ; 0.30] \quad 9.3 \%$

$0.00[0.00 ; 0.12] \quad 4.5 \%$

$0.02[0.00 ; 0.05] \quad 8.7 \%$

$0.15[0.02 ; 0.45] \quad 7.7 \%$

$0.00[0.00 ; 0.26] \quad 4.5 \%$

$0.00[0.00 ; 0.22] \quad 4.5 \%$

$0.04[0.01 ; 0.09] \quad 9.4 \%$

$0.14[0.03 ; 0.36] \quad 8.5 \%$

$0.31[0.09 ; 0.61] \quad 8.6 \%$

$0.12[0.06 ; 0.24] 100.0 \%$

FIG. 6. Forest plot for crude tumor control rate, crude endocrine remission rate, and any new hypopituitarism rate for prolactinoma. Squares indicate the proportions from individual studies and horizontal lines indicate the $95 \% \mathrm{Cl}$. The size of the data marker corresponds to the relative weight assigned in the pooled analysis using the random effects model. The diamond indicates the pooled proportion with $95 \% \mathrm{Cl}$. A: Forest plot for crude tumor control rate. B: Forest plot meta-analysis for crude endocrine remission rate. C: Forest plot for any new hypopituitarism rate. 


\section{TABLE 1. Recommendations for the use of SRS as part of the management of secretory PAs}

\begin{tabular}{l}
\hline Indications for SRS \\
Effective option to control growth of GH-, ACTH-, \& PRL-secreting residual or recurrent PAs after prior surgical resection but offers lower rate of \\
endocrine improvement or remission. \\
\hline Could be used as primary therapy for GH- \& ACTH-secreting PAs in patients deemed medically unfit for surgical resection. \\
Could be used as alternative to surgical resection for PRL-secreting PAs unresponsive to dopaminergic agonists. \\
Prescription dose \\
No minimal margin dose shown to definitively lead to better endocrine cure rates. Provided dose constraints safely protect surrounding structures \\
at risk (optic pathways, brainstem); higher margin doses can be used, although added therapeutic benefits in terms of remission remain not fully \\
defined. \\
SRS \& use of antisecretory drugs \\
Withdrawal of antisecretory medications preferred, typically for 4-12 wks prior to radiosurgery, if safely possible considering endocrinologic status \\
of patient. Timing of temporary cessation of antisecretory medications \& their reinstatement should be based on the particular agent pharmacology \\
\& patient's ability to tolerate brief withdrawal of medical management. \\
Endocrine remission \& tumor response criteria \\
Investigators should report outcomes using most recently accepted consensus criteria for response, from perspectives of tumor volume \& endo- \\
crine function.
\end{tabular}

mor size. Future studies should use 3D volumetric evaluations to report change in tumor dimensions after SRS, ideally using standardized criteria such as RECIST (Response Evaluation Criteria in Solid Tumours).

\section{Endocrine Remission After SRS}

The clinical presentation of secretory PA is most often related to the associated endocrinopathy rather than the compression of adjacent neural structures, and thus endocrine control is usually the main goal of management. Our meta-analysis confirms that SRS is more effective to control tumor growth than provide endocrine remission. Crude estimates for endocrine remission were $44 \%$ for acromegaly, $48 \%$ for Cushing's disease, and $28 \%$ for prolactinomas. These data must be interpreted with caution. First, most studies report crude endocrine remission rates at last follow-up, and very few report actuarial rates at different intervals. The biological effect of SRS on hormonal hypersecretion is believed to act over time. Therefore, crude rates might underestimate the true proportion of patients who will achieve remission after some latency period. For acromegaly, Pollock et al. reported cure rates of $11 \%$ at 2 years and $60 \%$ at 5 years after SRS. ${ }^{14}$ Similarly, Franzin et al. reported rates of $30.7 \%, 56.9 \%$, and $80.4 \%$ after 3, 5, and 10 years, respectively. ${ }^{7}$ Lee et al. reported cure in $31.7 \%$ after 2 years, increasing to $64.5 \%, 73.4 \%$, and $82.6 \%$ at 4,6 , and 8 years, respectively. ${ }^{5}$ Similar results were reported by Sheehan et al. in Cushing's disease patients, with cure in $34 \%$ at 1 year, $54 \%$ at 2 years, $72 \%$ at 3 years, and $78 \%$ at 5 years after SRS. ${ }^{34}$ In addition, the analyzed literature spans a 20-year interval, during which there have been changes in the criteria used to define endocrine remission. As recommended by most endocrinology societies, those criteria have become more refined in the recent years. The Cortina consensus defined acromegaly remission as a random $\mathrm{GH}$ level less than $1 \mathrm{ng} / \mathrm{ml}$ or nadir $\mathrm{GH}$ after an OGTT less than $0.4 \mathrm{ng} / \mathrm{ml}$ and normalization of age- and sex-adjusted IGF-1 levels. ${ }^{49}$ The more recent acromegaly consensus conference dropped the random $\mathrm{GH}$ level requirement and only kept the other two criteria (nadir GH after OGTT and normalization of IGF-1 levels). ${ }^{31}$ In this systematic review, only one of the selected studies used the more recent criteria to assess patients after SRS. In a series of 52 patients, Iwata et al. ${ }^{25}$ reported a crude cure rate of only $17 \%$. Using the older Cortina consensus criteria, Sala et al. ${ }^{24}$ achieved a cure in $41 \%$ of 22 patients included in their study. In comparison, by using $\mathrm{GH}$ levels less than $2.5 \mathrm{ng} / \mathrm{ml}$, Franzin et al. ${ }^{7}$ reported a cure rate of $60.7 \%$ and Yan et al. ${ }^{27}$ obtained a cure in $68.2 \%$ of patients. The same variability in endocrine cure definition is seen in Cushing's disease and prolactinoma studies. For Cushing's disease, normal 24-hour urinary free cortisol has been the most frequent criterion used. Alternatively, many authors used serum cortisol or ACTH levels, alone or in combination with urinary levels. For prolactinomas, most study investigators used normal PRL levels to define remission without specifying normal value ranges. Using more severe criteria will negatively impact cure rates, yet this is something that needs to be systematized moving forward.

\section{Hypopituitarism After SRS}

Among all included studies for all 3 secretory subtypes, new-onset or worsening of preexisting hypopituitarism was reported in $0 \%-50 \%$ of treated patients. Our metaanalysis failed to identify any significant factor associated with an increase in new hormone deficiency risk. No article included in the meta-analysis has demonstrated a correlation between endocrine remission and hypopituitarism, and we also could not find any such relation with the pooled data. We also could not find any relation between the margin dose and the risk of hypopituitarism. However, this lack of association might be more related to the poor quality of the included studies than a real lack of effect. As is the case for endocrine remission reporting, there is wide heterogenicity in the way studies report hypopituitarism (clinical vs biochemical confirmation, lack of pre-SRS hypopituitarism mention, lack of individual axis evaluation). In addition, because most studies only report crude hypo- 
pituitarism rates, this likely leads to an underestimation of the risk, as the rate of new hormone deficiency is likely to increase over time.

\section{Impact of the Prescription Dose}

PAs are usually benign in histology, and as such, single-fraction radiation doses of between 12 and 16 Gy have been demonstrated to provide durable control of tumor growth in NFA. ${ }^{48}$ This may also apply to hormone-secreting tumors, as crude tumor control rates between $83 \%$ and $100 \%$ have been reported across all 3 different studied subtypes, using median margin doses as low as 13-14 Gy in some studies. ${ }^{13,28}$ However, it has been widely believed that the doses required to stop the cellular mechanisms associated with hormonal hypersecretion are much higher than those required to control cellular division, and as such most authors have tried to deliver margin doses up to the range of 30-40 Gy, usually limited by the constraints of tumor volume and the proximity of optic apparatus. Despite this, only 2 of the selected studies actually found margin dose to be relevant for outcomes. In a study of 68 acromegalic patients, Zhang et al. found better tumor control and resolution of hypertension and hyperglycemia if margin doses were higher than $30 \mathrm{~Gy} .{ }^{21}$ Pan et al. reported improved chances of hyperprolactinemia resolution with margin doses of 30 Gy or more in 128 prolactinoma patients..$^{45}$ Our meta-regression analysis failed to demonstrate a relation between mean margin dose and crude endocrine remission rates for any of the 3 studies of tumor subtypes. However, this might only represent the fact that most authors have inherently used a dose selection threshold that is higher than the required one to produce an effect on hormone hypersecretion and might not be truly indicative of a lack of effect. In addition, regarding dose selection, the concept of a biologically effective dose (BED) might be more important for outcomes than the absolute dose. In a recent study of the Mayo Clinic acromegaly series (not included in this meta-analysis), BED remained the only factor correlated with endocrine remission on multivariate analysis. ${ }^{50}$ Regarding morbidity, we could not find any link between margin dose and the risk of pituitary function worsening among all tumor types, and the ophthalmological and neurological morbidity was low in the majority of studies, despite the high margin dose used. None of the included studies reported that increasing margin dose led to higher risk of visual or neurological morbidity. Lee et al. used a dose of 25 Gy in 136 acromegalic patients, ${ }^{5}$ with only $2.9 \%$ suffering from new visual field deficits and $0.7 \%$ new oculomotor palsy. Despite using 35 Gy, Jezková et al. did not report visual or neurological morbidity in 96 patients with acromegaly. ${ }^{16}$ Visual deterioration was reported in $11.1 \%$ of 71 patients in Kobayashi's acromegaly series, despite using a lower mean margin dose of 18.9 Gy. ${ }^{12}$ Based upon the available literature, if neuroanatomical dose constraints allow, higher margin doses could be utilized, although the added therapeutic benefit in terms of remission remains not fully defined.

\section{Impact of Antisecretory Medication}

The use of antisecretory drugs at the time of SRS is considered to negatively affect endocrine remission. For acromegaly, only the study by Pollock et al. confirmed this fact, with an HR of 4.2 of achieving remission when patients were off compared to on medication at SRS. ${ }^{14}$ Alternatively, 3 studies did not find medication intake to impact cure rates. ${ }^{5,15,17}$ It is worth noting that for the most recent acromegaly studies, suppressive medications were stopped for 1 to 4 months before SRS in all patients, making it difficult to confirm whether this is of importance or not. ${ }^{3,4,24,26,27}$ For prolactinomas, no study found a link between dopamine agonist intake and cure. Cohen-Inbar et al. reported that the use of a dopamine agonist at the time of SRS decreased cure rates from $50 \%$ in patients off medication to $38.1 \%$, but the difference failed to reach statistical significance. ${ }^{40}$ Two other studies mentioned no difference in endocrine remission based on medication intake at SRS. ${ }^{41,43}$ The impact of antisecretory medication is even less clear for Cushing's disease. The most common drug to control corticoid hypersecretion, ketoconazole, acts by decreasing peripheral cortisol synthesis at the adrenal level, although it might also reduce ACTH release from the anterior pituitary, as suggested by a preclinical study. ${ }^{51} \mathrm{In}$ the study by Sheehan et al., patients who were off ketoconazole at SRS had faster time to endocrine remission than patients on medication, but the overall endocrine remission rates were no different. ${ }^{34}$ Conversely, Castinetti et al. found statistically significantly better cure rates in patients off ketoconazole at the time of SRS (48\% vs 20\%). ${ }^{36}$ Taking all into consideration, it appears reasonable to recommend withholding antisecretory medication for 4 to 12 weeks (depending on the individual drug's pharmacokinetic profile) before SRS in patients in whom the endocrine status is stable and temporary drug cessation is not expected to cause any harmful deterioration.

\section{Timing of SRS in the Multimodality Management of Secretory PAs}

The management of secretory PAs frequently necessitates a combination of different modalities. SRS is usually viewed as a second or third-line option, after surgical or medical management. This is reflected in our meta-analysis, whereas the majority of included studies of acromegaly or Cushing's disease reported patients treated after surgical resection. Our results might not be applicable to patients who did not have prior resection. As such, we believe that patients who are deemed medically fit should be offered surgery as first-line management if possible. However, there was more heterogenicity in the prior management of patients who were included in our prolactinoma analyses, reflecting the fact that prolactinomas are exquisitely responsive to drug therapy, and surgery is rarely required for those patients. In addition, medically refractory prolactinomas tend to be more locally invasive, and surgery is generally deemed less likely to provide endocrine cure. In the study by Pan et al., only $2.3 \%$ of patients had prior surgical resection. ${ }^{45}$ Similarly, Jezková et al. reported resection in only $28.6 \%$ of patients before SRS. ${ }^{43}$ SRS seems to be used more liberally as second-line therapy and might be more appropriately recommended in this setting for prolactinomas than GH- or ACTH-secreting tumors. 


\section{Single-Fraction Versus Hypofractionated SRS}

In this meta-analysis, we deliberately chose to exclude fractionated stereotactic radiation therapy (SRT) studies and focused on SRS studies. Since the literature is already heterogeneous with respect to single-fraction SRS, the inclusion of the limited number of fractionated SRT studies would only add confounding factors to our study. Taking into account the accepted ISRS-endorsed definition of SRS, 2 hypofractionated SRS acromegaly studies that otherwise satisfied all inclusion criteria were included in the analysis. ${ }^{24,25}$ However, the results of those studies cannot be interpreted separately from the more prevalent singlefraction studies, so extrapolations of equivalency must be made with caution.

\section{Conclusions}

SRS offers effective tumor control of hormone-producing PAs in the majority of patients but a lower rate of endocrine improvement or remission. However, the quality of evidence that can be extracted from the existing literature on SRS for secretory PAs is low, with most studies being single-center retrospective studies in design. Limited practice recommendations can be formulated based on the evidence, and those recommendations are applicable only to the secretory subtypes included in this study (GH-, ACTH-, and PRL-secreting tumors) and should not be extrapolated to other histological subtypes.

\section{Acknowledgments}

We thank Prof. Frédéric Castinetti for his valuable comments on the manuscript.

\section{References}

1. Daly AF, Beckers A. The epidemiology of pituitary adenomas. Endocrinol Metab Clin North Am. 2020;49(3):347-355.

2. Moher D, Liberati A, Tetzlaff J, Altman DG. Preferred reporting items for systematic reviews and meta-analyses: the PRISMA statement. J Clin Epidemiol. 2009;62(10):1006-1012.

3. Kim EH, Oh MC, Chang JH, et al. Postoperative Gamma Knife radiosurgery for cavernous sinus-invading growth hormone-secreting pituitary adenomas. World Neurosurg. 2018;110:e534-e545.

4. Grant RA, Whicker M, Lleva R, et al. Efficacy and safety of higher dose stereotactic radiosurgery for functional pituitary adenomas: a preliminary report. World Neurosurg. 2014;82(12):195-201.

5. Lee CC, Vance ML, Xu Z, et al. Stereotactic radiosurgery for acromegaly. J Clin Endocrinol Metab. 2014;99(4):1273-1281.

6. Zeiler FA, Bigder M, Kaufmann A, et al. Gamma knife in the treatment of pituitary adenomas: results of a single center. Can J Neurol Sci. 2013;40(4):546-552.

7. Franzin A, Spatola G, Losa M, et al. Results of gamma knife radiosurgery in acromegaly. Int J Endocrinol. 2012;2012: 342034.

8. Liu X, Kano H, Kondziolka D, et al. Gamma knife radiosurgery for clinically persistent acromegaly. J Neurooncol. 2012; 109(1):71-79.

9. Castro DG, Cecílio SAJ, Canteras MM. Radiosurgery for pituitary adenomas: evaluation of its efficacy and safety. Radiat Oncol. 2010;5:109.

10. Iwai Y, Yamanaka K, Yoshimura M, et al. Gamma knife radiosurgery for growth hormone-producing adenomas. J Clin Neurosci. 2010;17(3):299-304.
11. Poon TL, Leung SCL, Poon CYF, Yu CP. Predictors of outcome following Gamma Knife surgery for acromegaly. $J$ Neurosurg. 2010;113(suppl):149-152.

12. Kobayashi T. Long-term results of stereotactic gamma knife radiosurgery for pituitary adenomas. Specific strategies for different types of adenoma. Prog Neurol Surg. 2009;22:77-95.

13. Swords FM, Monson JP, Besser GM, et al. Gamma knife radiosurgery: a safe and effective salvage treatment for pituitary tumours not controlled despite conventional radiotherapy. Eur J Endocrinol. 2009;161(6):819-828.

14. Pollock BE, Jacob JT, Brown PD, Nippoldt TB. Radiosurgery of growth hormone-producing pituitary adenomas: factors associated with biochemical remission. J Neurosurg. 2007; 106(5):833-838.

15. Vik-Mo EO, Oksnes M, Pedersen PH, et al. Gamma knife stereotactic radiosurgery for acromegaly. Eur J Endocrinol. 2007;157(3):255-263.

16. Jezková J, Marek J, Hána V, et al. Gamma knife radiosurgery for acromegaly-long-term experience. Clin Endocrinol (Oxf). 2006;64(5):588-595.

17. Castinetti F, Taieb D, Kuhn JM, et al. Outcome of gamma knife radiosurgery in 82 patients with acromegaly: correlation with initial hypersecretion. J Clin Endocrinol Metab. 2005;90(8):4483-4488.

18. Gutt B, Wowra B, Alexandrov R, et al. Gamma-knife surgery is effective in normalising plasma insulin-like growth factor I in patients with acromegaly. Exp Clin Endocrinol Diabetes. 2005;113(4):219-224.

19. Ikeda H, Jokura $H$, Yoshimoto T. Transsphenoidal surgery and adjuvant gamma knife treatment for growth hormone-secreting pituitary adenoma. J Neurosurg. 2001;95(2):285-291.

20. Izawa M, Hayashi M, Nakaya K, et al. Gamma knife radiosurgery for pituitary adenomas. J Neurosurg. 2000;93(suppl 3):19-22.

21. Zhang N, Pan L, Wang EM, et al. Radiosurgery for growth hormone-producing pituitary adenomas. J Neurosurg. 2000; 93(suppl 3):6-9.

22. Mokry M, Ramschak-Schwarzer S, Simbrunner J, et al. A six year experience with the postoperative radiosurgical management of pituitary adenomas. Stereotact Funct Neurosurg. 1999;72 Suppl 1(1):88-100.

23. Landolt AM, Haller D, Lomax N, et al. Stereotactic radiosurgery for recurrent surgically treated acromegaly: comparison with fractionated radiotherapy. J Neurosurg. 1998;88(6): 1002-1008.

24. Sala E, Moore JM, Amorin A, et al. CyberKnife robotic radiosurgery in the multimodal management of acromegaly patients with invasive macroadenoma: a single center's experience. J Neurooncol. 2018;138(2):291-298.

25. Iwata H, Sato K, Nomura R, et al. Long-term results of hypofractionated stereotactic radiotherapy with CyberKnife for growth hormone-secreting pituitary adenoma: evaluation by the Cortina consensus. J Neurooncol. 2016;128(2):267-275.

26. Wilson PJ, De-Loyde KJ, Williams JR, Smee RI. Acromegaly: a single centre's experience of stereotactic radiosurgery and radiotherapy for grow th hormone secreting pituitary tumours with the linear accelerator. J Clin Neurosci. 2013; 20(11):1506-1513.

27. Yan JL, Chang CN, Chuang CC, et al. Long-term follow-up of patients with surgical intractable acromegaly after linear accelerator radiosurgery. J Formos Med Assoc. 2013;112(7): 416-420.

28. Sallabanda K, Usychkin S, Puebla F, et al. Stereotactic radiosurgery in pituitary adenomas: long-term single institution experience and role of the hypothalamic-pituitary axis. $J$ Radiosurg SBRT. 2011;1(3):213-220.

29. Voges J, Kocher M, Runge M, et al. Linear accelerator radiosurgery for pituitary macroadenomas: a 7-year follow-up study. Cancer. 2006;107(6):1355-1364. 
30. Wan H, Chihiro O, Yuan S. MASEP gamma knife radiosurgery for secretory pituitary adenomas: experience in 347 consecutive cases. J Exp Clin Cancer Res. 2009;28:36.

31. Melmed S, Bronstein MD, Chanson P, et al. A Consensus Statement on acromegaly therapeutic outcomes. Nat Rev Endocrinol. 2018;14(9):552-561.

32. Narayan V, Mohammed N, Bir SC, et al. Long-term outcome of nonfunctioning and hormonal active pituitary adenoma after Gamma Knife radiosurgery. World Neurosurg. 2018; 114:e824-e832.

33. Marek J, Ježková J, Hána V, et al. Gamma knife radiosurgery for Cushing's disease and Nelson's syndrome. Pituitary. 2015;18(3):376-384.

34. Sheehan JP, Xu Z, Salvetti DJ, et al. Results of Gamma Knife surgery for Cushing's disease. J Neurosurg. 2013;119(6): 1486-1492.

35. Tinnel BA, Henderson MA, Witt TC, et al. Endocrine response after Gamma Knife-based stereotactic radiosurgery for secretory pituitary adenoma. Stereotact Funct Neurosurg. 2008;86(5):292-296.

36. Castinetti F, Nagai M, Dufour H, et al. Gamma knife radiosurgery is a successful adjunctive treatment in Cushing's disease. Eur J Endocrinol. 2007;156(1):91-98.

37. Wilson PJ, Williams JR, Smee RI. Cushing's disease: a single centre's experience using the linear accelerator (LINAC) for stereotactic radiosurgery and fractionated stereotactic radiotherapy. J Clin Neurosci. 2014;21(1):100-106.

38. Wein L, Dally M, Bach LA. Stereotactic radiosurgery for treatment of Cushing disease: an Australian experience. Intern Med J. 2012;42(10):1153-1156.

39. Devin JK, Allen GS, Cmelak AJ, et al. The efficacy of linear accelerator radiosurgery in the management of patients with Cushing's disease. Stereotact Funct Neurosurg. 2004;82(5-6): 254-262.

40. Cohen-Inbar O, Xu Z, Schlesinger D, et al. Gamma Knife radiosurgery for medically and surgically refractory prolactinomas: long-term results. Pituitary. 2015;18(6):820-830.

41. Liu X, Kano H, Kondziolka D, et al. Gamma knife stereotactic radiosurgery for drug resistant or intolerant invasive prolactinomas. Pituitary. 2013;16(1):68-75.

42. Tanaka S, Link MJ, Brown PD, et al. Gamma knife radiosurgery for patients with prolactin-secreting pituitary adenomas. World Neurosurg. 2010;74(1):147-152.

43. Jezková J, Hána V, Krsek M, et al. Use of the Leksell gamma knife in the treatment of prolactinoma patients. Clin Endocrinol (Oxf). 2009;70(5):732-741.

44. Petrovich Z, Yu C, Giannotta SL, et al. Gamma knife radiosurgery for pituitary adenoma: early results. Neurosurgery. 2003;53(1):51-61.

45. Pan L, Zhang N, Wang EM, et al. Gamma knife radiosurgery as a primary treatment for prolactinomas. J Neurosurg. 2000; 93(suppl 3):10-13.

46. Wilson PJ, Williams JR, Smee RI. Single-centre experience of stereotactic radiosurgery and fractionated stereotactic radiotherapy for prolactinomas with the linear accelerator. $J$ Med Imaging Radiat Oncol. 2015;59(3):371-378.

47. Yoon SC, Suh TS, Jang HS, et al. Clinical results of 24 pituitary macroadenomas with linac-based stereotactic radiosurgery. Int J Radiat Oncol Biol Phys. 1998;41(4):849-853.

48. Kotecha R, Sahgal A, Rubens M, et al. Stereotactic radiosurgery for non-functioning pituitary adenomas: meta-analysis and International Stereotactic Radiosurgery Society practice opinion. Neuro Oncol. 2020;22(3):318-332.

49. Giustina A, Chanson P, Bronstein MD, et al. A consensus on criteria for cure of acromegaly. J Clin Endocrinol Metab. 2010;95(7):3141-3148.
50. Graffeo CS, Donegan D, Erickson D, et al. The impact of insulin-like growth factor index and biologically effective dose on outcomes after stereotactic radiosurgery for acromegaly: cohort study. Neurosurgery. 2020;87(3):538-546.

51. Stalla GK, Stalla J, Loeffler JP, et al. Pharmacological modulation of CRH-stimulated ACTH secretion by ketoconazole. Horm Metab Res Suppl. 1987;16:31-36.

\section{Disclaimer}

These guidelines should not be considered inclusive of all methods of care or exclusive of other methods or care reasonably directed to obtain similar results. The physician must make the ultimate judgment depending on characteristics and circumstances of individual patients. Adherence to this guideline will not ensure successful treatment in every situation. The authors of this guideline and the International Society of Stereotactic Radiosurgery assume no liability for the information, conclusions, and recommendations contained in this report.

\section{Disclosures}

R. Kotecha: honoraria from Elsevier, Elekta AB, Accuray Inc., Novocure, and Viewray Inc.; member of speaker's bureau for Novocure Inc. A. Sahgal: advisor/consultant with Abbvie, Merck, Roche, Varian (Medical Advisory Group), Elekta (Gamma Knife Icon), Brainlab, and VieCure (Medical Advisory Board); ex officio board member of International Stereotactic Radiosurgery Society (ISRS); past educational seminars with Elekta AB, Accuray Inc., Varian (CNS Teaching Faculty), Brainlab, and Medtronic Kyphon; research grant with Elekta AB; travel accommodations/expenses by Elekta, Varian, and Brainlab, also belongs to the Elekta MR LINAC Research Consortium, Elekta Spine, Oligometastases, and LINAC-based SRS consortia. I. Paddick: consulting work with Elekta AB. J. Régis: consultant for Elekta; J. Suh: advisor/consultant for Philips, Novocure, and Neutron Therapeutics.

\section{Author Contributions}

Conception and design: Mathieu, Kotecha, Sahgal, Sheehan. Acquisition of data: Mathieu. Analysis and interpretation of data: Mathieu, Kotecha, Rubens, Sheehan. Drafting the article: Mathieu. Critically revising the article: all authors. Reviewed submitted version of manuscript: all authors. Approved the final version of the manuscript on behalf of all authors: Mathieu. Statistical analysis: Kotecha, Rubens. Study supervision: Mathieu, Sahgal, Sheehan.

\section{Supplemental Information \\ Online-Only Content}

Supplemental material is available with the online version of the article.

Supplementary Figures and Tables. https://thejns.org/doi/ suppl/10.3171/2021.2.JNS204440.

\section{Previous Presentations}

Presented in part at the 14th International Stereotactic Radiosurgery Society Congress in Rio de Janeiro, Brazil, June 9-13, 2019.

\section{Correspondence}

David Mathieu: Université de Sherbrooke, Centre de recherche du Centre Hospitalier Universitaire de Sherbrooke, Sherbrooke, QC, Canada.david.mathieu@usherbrooke.ca. 